\title{
A Multi Polarization Square Patch Antenna with a Reconfigurable Feeding Network
}

\author{
Roozbeh Rezaeipour, Ramezanali Sadeghzadeh
}

\begin{abstract}
A multi-polarization square patch antenna with a reconfigurable feeding network is presented in this paper. The reconfigurable feeding network of this antenna is implemented on an FR-4 substrate by a Wilkinson power divider and a branch line coupler which perform amplitude distribution in the feeding network. Besides, two switching circuits, which consist of one PIN diode (BAR63-02w) and its DC biasing circuit, manage the RF signal flow on this feeding network. These switching circuits control the phase of the RF signal applied to the square patch, so it can provide linear polarization, left-hand and right-hand circular polarization at $2.45 \mathrm{GHz}$, which has many applications in wireless networks. The simulated and measured results are presented, which illuminate acceptable axial ratio bandwidth (ARBW) for both right-hand and left-hand circular polarization in $(2.38-2.48 \mathrm{GHz})$ and minimum $-10 \mathrm{~dB}$ return loss at $2.45 \mathrm{GHz}$.
\end{abstract}

Index Terms-Multi-Polarization, Reconfigurable antenna, PIN-Diode, Power divider

\section{INTRODUCTION}

$\mathbf{M}$ ULTI-polarization antenna has appealed to attentions since compact wireless systems were expanded. These antennas are also known as "polarization agile antenna", used in Wireless Local Area Network (WLAN), microwave tagging systems, and diversity polarization. Their characteristics can be altered in real-time and be capable of communication system considerations. Hence, they have a lightweight and compact size which is essential for wireless systems. Furthermore, reusing of the frequency spectrum and doubled capacity are satisfied in such designs [1][2]. Multi-polarization can relieve signal losing led by multipath effect. It can be implemented in two ways: using reconfigurable radiation elements or designing suitable feeding networks [3].

The reconfigurability is implemented in the main characteristics of an antenna by manipulating the current distribution over the antenna element or feeding network structure. A variety of methods are utilized for the reconfigurable antenna. [4] - [17]. In [4], dual-frequency operation is satisfied by locating varactors and capacitors in slots. The second resonant frequency can be tuned by changing the DC voltage. Also in [5], a hybrid reconfigurable antenna is introduced which frequency reconfigurability is realized by controlling the states of switch electronically. Moreover, a pattern-reconfigurable antenna is the other way of reusing common structures of an antenna that is explored in [6], [7]. In [6], the patternreconfigurable antenna is achieved over a square-ring patch antenna by placing four shorting walls and controlling two numbers of walls by applying DC voltage so the antenna can operate in two modes: monopolar plat-patch and normal patch modes. Hence, the antenna's radiation pattern can be switched between conical and broadside radiations pattern at a specific frequency. Additionally, polarization reconfigurable antennas based on two ways of implementation are presented in [8][16].

Using active microwave elements such as PIN diodes, varactors, RF switching circuits, or power dividers are frequent in polarization reconfigurable antenna designing. Two methods are employed for this type of antenna, which is using the active component as a part of radiation elements of antenna structure or using them in feeding network. In both methods, switches can be controlled in ON and OFF states by a DC controlling circuit [9]. In [8], two slot-ring with perturbations antenna is presented which can switch between two CP and one LP by creating discontinuities at a specific angle and placing PIN diode for switching polarization. Perturbation is used in [2] on a square-ring slot. Small conducting pads are connected by a PIN diode, and multi-polarization is implemented through diode switching. In [10], two PIN diodes are added in the optimized location of the E-shaped antenna structure. The antenna has a simple structure that is capable of switching its polarization between RHCP and LHCP. In [11], a simple microstrip patch antenna with a slot fed by a coaxial diagonally is probed. Pin diode is in charge of adjusting $\mathrm{CP}$ polarization in different frequencies by two capacitors and a DC controlling circuit. In [12], [13], the same structure is introduced with one and two feed lines, respectively. In a square patch antenna, circular polarization is realized by truncating corners of the patch [14]. By placing pin diodes with their DC bias on cut corners multi-polarization is achieved on this structure. Altering in feeding networks is performed in [15]-[16]. In [15], multi-polarization is designed by a four-way power divider to feed the antenna. When all patches are excited with equal phases and amplitudes linear polarization is radiated, whereas equal amplitudes and 90 degrees phase differences for each output conducts circular polarization. Although in [17] and [16] feeding networks are sophisticated, all structures led to a multi-polarization antenna with four polarizations. PIN diodes are used in feeding network design, and their DC controlling block can select suitable polarization for radiating at 2.4 GHz.All mentioned antennas utilize RF components in their structure for achieving reconfigurability.

In this paper, a reconfigurable feeding network is implemented by two types of power dividers and pairs of PIN diodes. Power dividers perform dividing power applied to the input port of the antenna equally between two ports connected to the microstrip patch antenna. Pairs of PIN diodes and their DC controlling circuits manage the phase of the RF signal going through a branch line coupler to the microstrip 


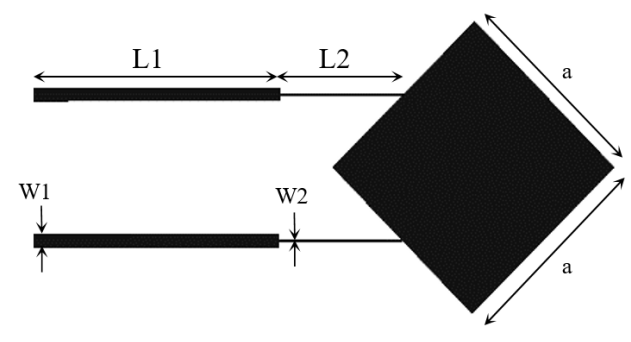

Fig. 1: Structure of the antenna

TABLE I: Dimension of Proposed Antenna in mm

\begin{tabular}{|c|c|c|c|c|}
\hline $\mathrm{a}$ & $\mathrm{W} 1$ & $\mathrm{~L} 1$ & $\mathrm{~W} 2$ & $\mathrm{~L} 2$ \\
\hline 27 & 1.84 & 36 & 0.26 & 18 \\
\hline
\end{tabular}

patch antenna. As a result, a multi-polarization antenna will be realized on this structure. The desirable antenna structure with its feeding network and explanation of the components' function is introduced in section II. Then, in the section III results of the simulation done by CST are presented. Moreover, experimental results are presented in IV. Finally, section V is the conclusion.

\section{Antenna Configuration and Design Process}

\section{A. Antenna Configuration}

Technically, achieving circular polarization on a microstrip patch antenna is based on two factors: equal amplitude and 90 degrees phase difference between two orthogonal modes of the antenna. Moreover, there are many methods for realizing circular polarization on microstrip patch antenna rooted in factors mentioned above [18]. In this paper, a square microstrip patch antenna (Fig. 1) is designed for $2.45 \mathrm{GHz}$ and fabricated on an FR4 substrate with $1 \mathrm{~mm}$ height and 4.6 permittivity. Circular polarization is implemented by exciting central points of two edges of the square microstrip patch antenna by equal amplitude and 90 degrees phase differences. As a result, TM01 and TM10 modes of this structure are excited with 90 degrees phase difference leading to radiate circular polarization (CP). In addition, linear polarization (LP) is accomplished on the same design by feeding the square patch antenna with identical amplitude without any phase differences. The parameters and dimensions of the antenna and matching lines are handed in Table I and II, respectively.

\section{B. Feeding Network}

Power dividers are broadly used in feeding networks of the antennas. In this paper, a Wilkinson power divider (Fig. 2) and a branch line coupler (Fig. 3) are utilized as feeding network of the proposed antenna. Parameters and dimensions of these power dividers are given in Table II and III, respectively. Feeding network of this antenna is designed for $2.45 \mathrm{GHz}$ and constructed on an FR-4 substrate with 4.6 permittivity. In the first part of this feeding network, an RF signal applied to the Wilkinson power divider leads to equal phases and amplitudes at output ports of this power divider. In the second part of this feeding network, the RF signal enters the branch line coupler

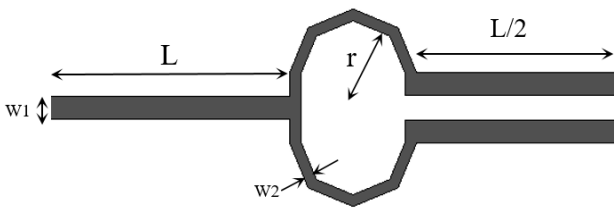

Fig. 2: Structure for the Wilkinson power divider

TABLE II: Dimension for the Wilkinson power divider in $\mathrm{mm}$

\begin{tabular}{|c|c|c|c|}
\hline $\mathrm{r}$ & $\mathrm{L}$ & $\mathrm{W} 1$ & $\mathrm{~W} 2$ \\
\hline 4.72 & 36 & 1.84 & 0.96 \\
\hline
\end{tabular}

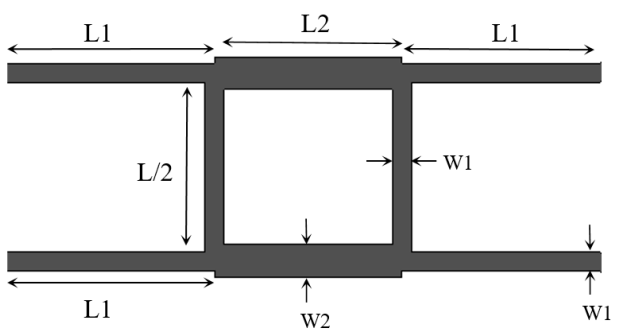

Fig. 3: Structure of the branch line coupler

TABLE III: Dimension of the branch line coupler in mm

\begin{tabular}{|c|c|c|c|}
\hline L1 & W1 & L2 & W2 \\
\hline 36 & 1.84 & 16.16 & 0.96 \\
\hline
\end{tabular}

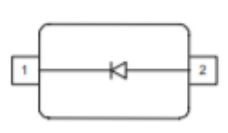

(a)

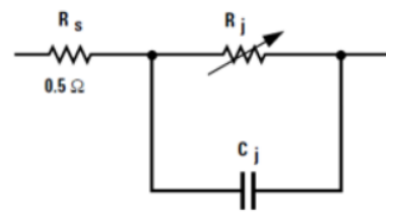

(b)
Fig. 4: PIN diode (a) Schematic (b) Equivalent circuit [19]

which can turn applied power into two RF signals with equal amplitude and 90 degrees phase difference. Consequently, if mentioned antenna in section A. is joined to this feeding network, circular polarization's conditions will be satisfied. On the other hand, if both ports of the branch line coupler are excited, the antenna will be fed by an RF signal with equivalent phase and amplitude which is indispensable for linear polarization.

\section{Operation Mechanism}

In this part, polarization of the proposed antenna according to the excited ports of the branch line coupler is discussed. Polarization control is undertaken by two single PIN diodes (BAR64-02w) (Fig. 4). These PIN diodes are placed in the output ports of the Wilkinson power divider which are connected to the input ports of the branch line coupler in the final designed structure of the antenna Fig. 5. PIN diodes control the RF signal flow toward the branch line coupler determining exciting phase and amplitude of the antenna. The equivalent circuit of the PIN diode according to the 


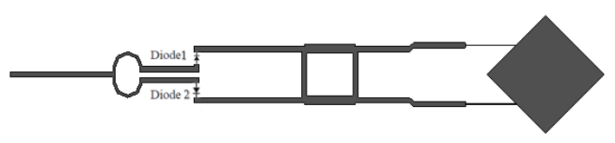

Fig. 5: Final structure of proposed antenna

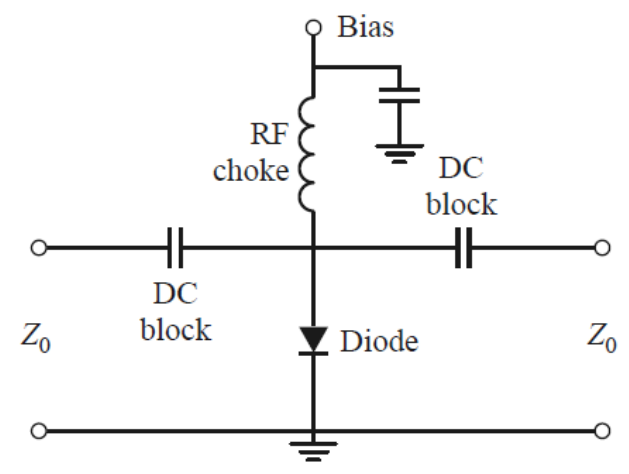

Fig. 6: Schematic of switching circuit [18]

company's datasheet is depicted in Fig. 4 [19]. The variable resistor ( $\mathrm{Rj}$ ) determines the ON or OFF state of the PIN diode controlled by the DC biasing circuit. As a result, biasing current provides access to ON and OFF states of the PIN diodes by changing the variable resistor. Basically, according to the equivalent circuit of the PIN diode, PIN diode switching circuit is realized in two configurations: (1) Series, (2) Shunt [18]. In this paper, the shunt configuration of the PIN diode with biasing circuit in Fig. 6 is utilized. A constant 45 -ohm resistor is fixed at the input port of both switching circuits. The switching circuit can provide just over $-3 \mathrm{~dB}$ insertion loss in OFF state and $-14 \mathrm{~dB}$ isolation in ON state which is appropriate for adjusting the RF signal flow applied to square patch antenna for achieving different operation modes. These two switching circuits are located at the input ports of the branch line coupler. Consequently, according to the configuration of the switches, if two PIN diodes are OFF, both branch line coupler's inputs are excited by the applied RF signal which leads to the same phases and amplitude in inputs of the square patch antenna. So, linear polarization will be radiated at the designed frequency. Besides, if diode 1 is $\mathrm{OFF}$ and 2 is ON, only one input of the branch line coupler is excited which makes 90 degrees phase difference between the two excited modes of the square patch antenna. So, in this operation mode Left Hand Circular Polarization (LHCP) is radiated at the designed frequency. Also this structure can radiate Right Hand Circular Polarization (RHCP). If diode 1 is $\mathrm{ON}$ and diode 2 is OFF, unlike the previous state, phase difference and identical amplitude are prepared reversely on input ports of the branch line coupler which leads to RHCP in this operation mode. As a result, the antenna can radiate three different polarizations with one feed port on the same structure. Operation modes of the antenna with states of the PIN diodes are shown in Table IV.
TABLE IV: Antenna Polarization

\begin{tabular}{|c|c|c|c|}
\hline Polarization & LP (Ant.1) & LHCP (Ant.2) & RHCP (Ant.3) \\
\hline Diode 1 & OFF & OFF & ON \\
\hline Diode 2 & OFF & ON & OFF \\
\hline
\end{tabular}

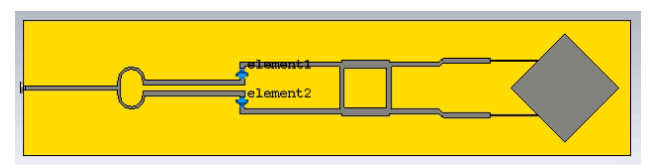

Fig. 7: Schematic of antenna in CST simulation

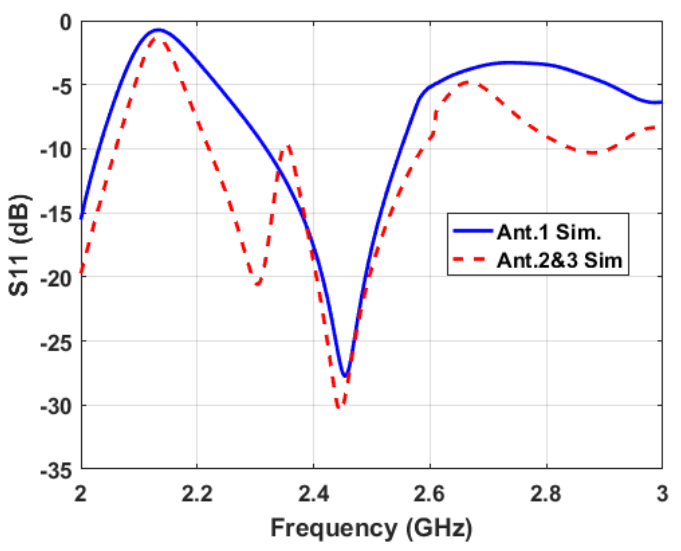

Fig. 8: Simulated return loss for Ant.1

\section{DC Biasing}

A shunt DC biasing circuit shown in Fig. 6 is utilized in the switching circuit of this reconfigurable patch antenna. Two $47 \mathrm{nF}$ capacitors in the 0402 standard size package are used in the RF input and output of the switching circuit. These capacitors prevent leaking DC voltage to the RF source. An external voltage supply makes a $5 \mathrm{~mA}$ current to change the state of the PIN diode. This voltage is applied through a $22 \mathrm{nH}$ inductance in the 0402 standard size package blocking RF leakage into the DC voltage supply. The DC voltage supply with a $47 \mathrm{nF}$ grounded capacitor in the 0402 standard package is connected to each switching circuit through two vias placed underneath the antenna structure. Also, The applied voltage can be controlled by two mechanical switches which are placed separately in an external box for better access.

\section{Simulation Result}

The primary implementation of the discussed structure in the previous section is performed and simulated in the layout part of the Advanced Design System (ADS) software. Then, it is converted to the CST software (Fig. 7) for validating and checking the radiation characteristics. DC analysis and simulation of switching circuits in ON and OFF states which were mentioned in B. Operating mechanism is done by the ADC software then the touchstone file is exported to be utilized in further simulation. In the CST simulation, the mentioned touchstone files of switching circuits for all operation modes of the antenna are placed in the schematic part of the CST (Fig.7) 


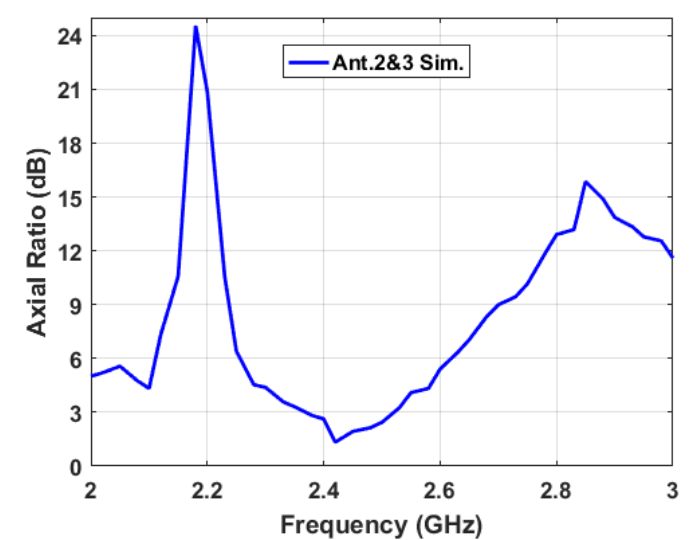

Fig. 9: Simulated axial ratio for Ant.2 and Ant.3

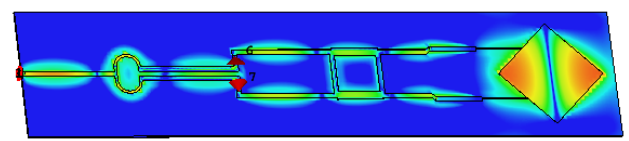

(a) Linear polarization for (Ant.1)

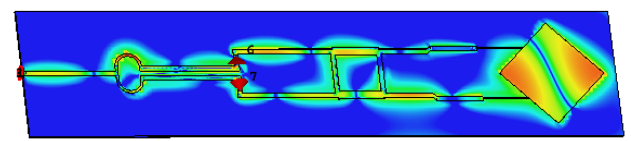

(b) Right hand circular polarization (LHCP) for (Ant.2)

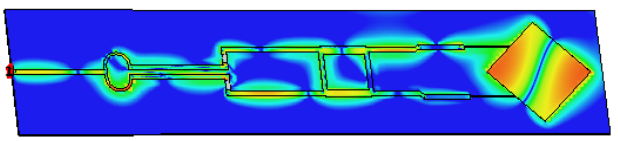

(c) Left hand circular polarization (RHCP) for (Ant.3)

Fig. 10: Simulated electrical field distribution for all operation modes

for attaining the radiation characteristics in CST results. The simulated return loss of Ant.1 is shown in Fig 8. The minimum simulated return loss in the linear polarization state (Ant.1) is $-27.7 \mathrm{~dB}$ at $2.45 \mathrm{GHz}$ and the impedance bandwidth is 9.5\%. The return loss of Ant.2 and Ant. 3 are drawn in Fig. 8. These reflection coefficients are equal since the structure of the antenna is symmetrical. The minimum simulated return loss in the Light Hand Circular polarization LHCP (Ant.2) and the Right Hand Circular Polarization state RHCP (Ant.3) is $-30.4 \mathrm{~dB}$ at $2.44 \mathrm{GHz}$ and the impedance bandwidth is 9\%. As a result, the operating frequency is identical for all antennas on the same structure. Moreover, the simulated impedance bandwidth in all three antennas is $200 \mathrm{MHz}(9 \%)$ which is acceptable for wireless applications. Radiating circular polarization is necessarily having below $3 \mathrm{~dB}$ axial ratio (AR) in both left hand (Ant.2) or right hand (Ant.3) circular polarization. The simulated axial ratio (AR) for Ant.2 and Ant.3 is drawn in Fig. 9. Due to the symmetrical structure of this antenna, both antennas have the same axial ratio in the simulation result. The minimum simulated axial ratio is $1 \mathrm{~dB}$ at $2.43 \mathrm{GHz}$ and the axial ratio bandwidth (ARBW) is $4.5 \%$. What determines the polarization of the antenna which can

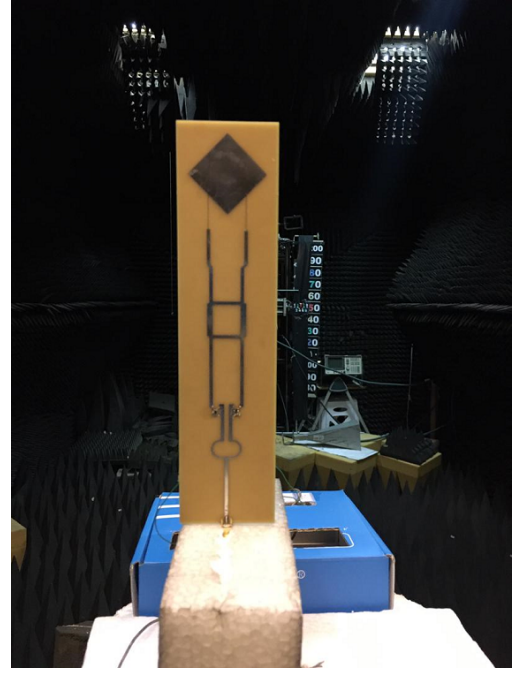

Fig. 11: Antenna in testing conditions

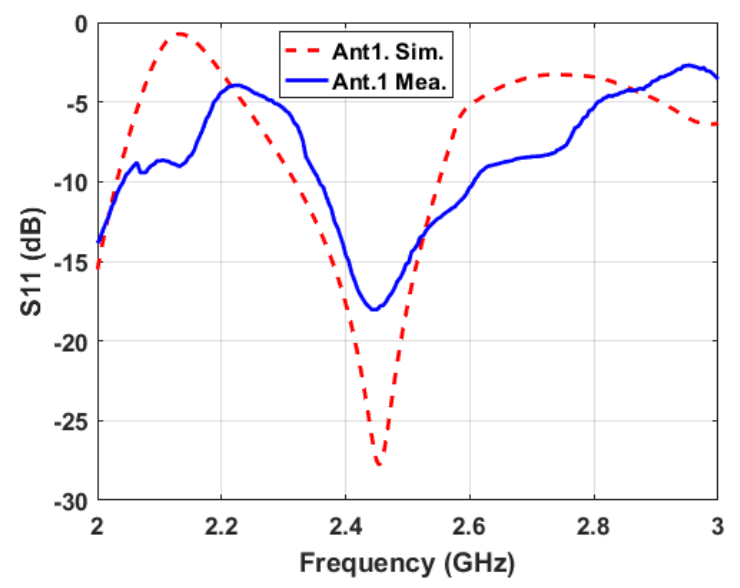

Fig. 12: Simulated and measured return loss for Ant.1

be controlled by switching circuits, is adjusting the form of the current distribution. It can make 0 and 90 degrees phase difference in E-field distribution which is applied to square patch and causes linear polarization and circular polarization respectively. The E-field distributions related to each antenna are depicted in Fig. 10 (a)-(c). Optically, the same phase excitation is seen in Fig. 10 (a) which is related to the linear polarization Ant.1. In addition, 90 degrees phase difference in Left-hand Circular Polarization (LHCP) and Right-Hand Circular Polarization (RHCP) is extracted in Fig. 12 (b) and 12 (c), respectively.

\section{EXPERIMENTAL RESULT}

In this section, experimental and simulation results will be discussed. Fig. 11 exhibits the designed antenna prototype in testing conditions fabricated by the printed circuit board (PCB) technology and its external DC controlling switches.

\section{A. Matching}

The measured and simulated reflection coefficient for Ant.1 is drawn in Fig. 12. Linear polarization in both results has the 


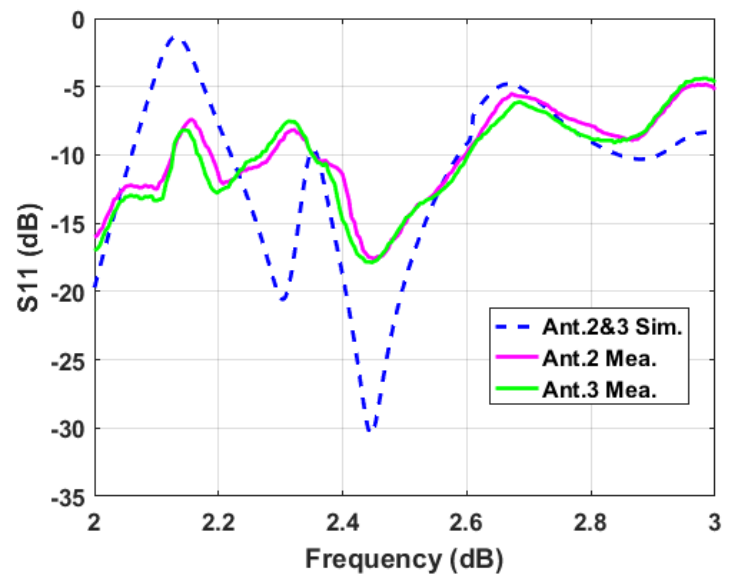

Fig. 13: Simulated and measured return loss for Ant.2 and 3

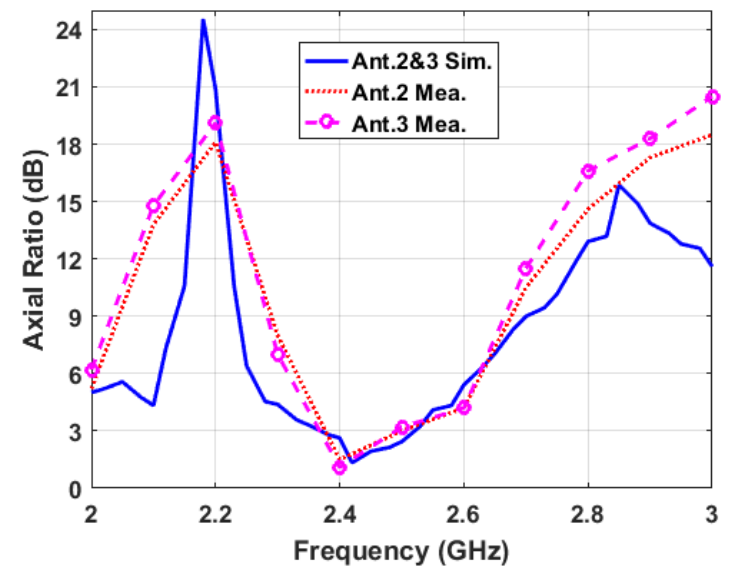

Fig. 14: Simulated and measured axial ratio for Ant.2 and Ant.3

same $-10 \mathrm{~dB}$ bandwidth and operating frequency. The $-10 \mathrm{~dB}$ bandwidth for simulated is $8 \%$ and the minimum return loss in this antenna is at $2.45 \mathrm{GH}$. Moreover, measured and simulated reflection coefficient for LHCP(Ant.2) and RHCP(Ant.3) state of this antenna are depicted in Fig. 13. Return loss in these circular polarization operation modes of this antenna has the same $-10-\mathrm{dB}$ bandwidth and operating frequency of $8 \%$ and $2.45 \mathrm{GHz}$, respectively. Consequently, comparing measured return loss of all possible states of this antenna implies that this antenna has satisfactory impedance matching in all possible states at designed frequency.

\section{B. Radiation Characteristics}

The measured and the simulated axial ratio of the Ant.2 (LHCP) and the Ant.3 (RHCP) are illustrated in Fig. 14. All measured results are done in the main lobe of the antenna pattern (theta $=30, \mathrm{Phi}=0$ ) and $200 \mathrm{MHz}$ steps are used for obtaining measured results. The measured axial ratio bandwidth (ARBW) of Ant.2 and Ant.3 are (2.39-2.48 GHz) 3.63\% and $(2.36-2.47 \mathrm{GHz}) 4.4 \%$, respectively. Thus, this antenna can radiate left-hand and right-hand circular polarization on (a)

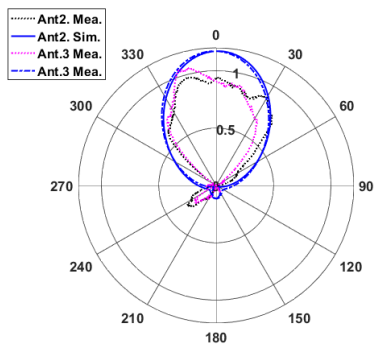

(c)

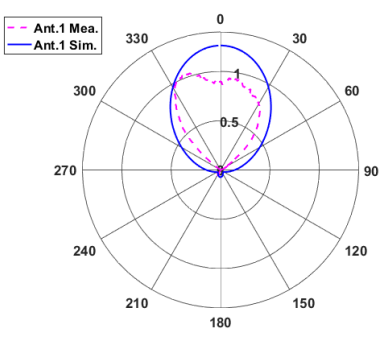

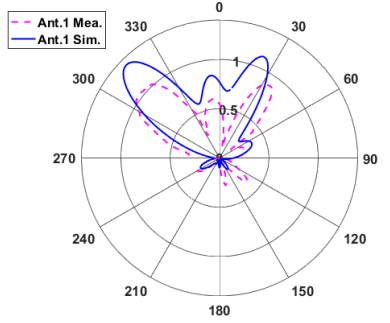

(b)

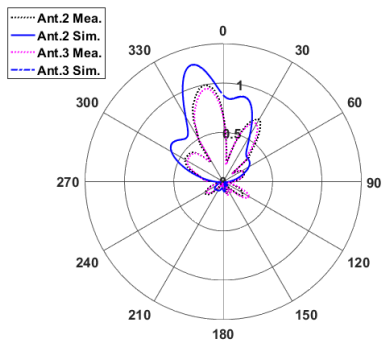

(d)
Fig. 15: Simulated and measured radiation pattern at $2.45 \mathrm{GHz}$ of (a) LP in H-Plane (b) LP in E-Plane (c) LHCP and RHCP in H-Plane (d) LHCP and RHCP in E-Plane

the same structure at designed frequency. Likewise, the slight discrepancies between the simulated and measured are rooted in the small tolerance of the fabrication process. As a result of the axial ratio and reflection coefficient, this antenna can perform at three different polarizations in a specific frequency according to the overlap of axial ratio bandwidth and $-10 \mathrm{~dB}$ bandwidth in all possible states of the antenna.

Normalized radiation patterns of this antenna in all operation modes are illustrated in Fig. 15. Measured radiation patterns in E-plane and H-plane for LHCP and RHCP modes of this antenna are approximately equal due to the symmetrical antenna structure. All directional patterns of operation modes are achieved with $1.2 \mathrm{dBi}, 1 \mathrm{dBi}$, and $0.98 \mathrm{dBi}$ gain for Ant.1, Ant.2, and Ant.3, respectively. Consequently, this antenna can perform at three different polarization at a specific frequency according to the overlap of axial ratio bandwidth and $-10 \mathrm{~dB}$ bandwidth in all possible antenna states.

\section{CONCLUSION}

A triple-polarization square patch antenna has been presented in this paper. Polarization reconfigurability with the same feeding port is achieved by a combining of two power dividers and two PIN diodes as switching circuits. These switching circuits control the RF signal flow applied to the power dividers and the square patch antenna. Additionally, polarization switching can be handled by changing the DC voltage of the shunt biasing circuits of PIN diodes. The impedance bandwidth coincides with the axial ratio's bandwidth according to the measured results. So, this design considers all requirements for wireless applications at 2.45 GHz. 


\section{REFERENCES}

[1] A. Khidre, K.-F. Lee, F. Yang, and A. Z. Elsherbeni, "Circular polarization reconfigurable wideband e-shaped patch antenna for wireless applications," IEEE transactions on antennas and propagation, vol. 61, no. 2, pp. 960-964, 2012.

[2] M. K. Fries, M. Grani, and R. Vahldieck, "A reconfigurable slot antenna with switchable polarization," IEEE Microwave and Wireless Components Letters, vol. 13, no. 11, pp. 490-492, 2003.

[3] W. Lin and H. Wong, "Wideband circular polarization reconfigurable antenna," IEEE Transactions on Antennas and Propagation, vol. 63, no. 12, pp. 5938-5944, 2015.

[4] N. Behdad and K. Sarabandi, "Dual-band reconfigurable antenna with a very wide tunability range," IEEE Transactions on Antennas and Propagation, vol. 54, no. 2, pp. 409-416, 2006.

[5] L. Ge, "A frequency, polarization and radiation pattern reconfigurable slot antenna," in 2017 International Workshop on Electromagnetics: Applications and Student Innovation Competition. IEEE, 2017, pp. 23-25.

[6] S.-H. Chen, J.-S. Row, and K.-L. Wong, "Reconfigurable square-ring patch antenna with pattern diversity," IEEE Transactions on Antennas and Propagation, vol. 55, no. 2, pp. 472-475, 2007.

[7] P.-Y. Qin, Y. J. Guo, Y. Cai, E. Dutkiewicz, and C.-H. Liang, "A reconfigurable antenna with frequency and polarization agility," IEEE antennas and wireless propagation letters, vol. 10, pp. 1373-1376, 2011.

[8] W. M. Dorsey, A. I. Zaghloul, and M. G. Parent, "Perturbed squarering slot antenna with reconfigurable polarization," IEEE Antennas and Wireless Propagation Letters, vol. 8, pp. 603-606, 2009.

[9] W. Lin and H. Wong, "Polarization reconfigurable wheel-shaped antenna with conical-beam radiation pattern," IEEE Transactions on Antennas and Propagation, vol. 63, no. 2, pp. 491-499, 2014.

[10] A. Khidre, K.-F. Lee, F. Yang, and A. Z. Elsherbeni, "Circular polarization reconfigurable wideband e-shaped patch antenna for wireless applications," IEEE transactions on antennas and propagation, vol. 61, no. 2, pp. 960-964, 2012.

[11] N. Jin, F. Yang, and Y. Rahmat-Samii, "A novel patch antenna with switchable slot (pass): Dual-frequency operation with reversed circular polarizations," IEEE Transactions on Antennas and Propagation, vol. 54 no. 3, pp. 1031-1034, 2006.

[12] Y. Sung, T. Jang, and Y.-S. Kim, "A reconfigurable microstrip antenna for switchable polarization," IEEE microwave and wireless components letters, vol. 14, no. 11, pp. 534-536, 2004.

[13] M. Y. Al-mallah, Z. I. Saleh, and Y. S. Faouri, "Two feed reconfigurable microstrip patch antenna for polarization diversity," in 2019 IEEE Jordan International Joint Conference on Electrical Engineering and Information Technology (JEEIT). IEEE, 2019, pp. 118-120.

[14] W.-S. Chen, C.-K. Wu, and K.-L. Wong, "Novel compact circularly polarized square microstrip antenna," IEEE Transactions on Antennas and propagation, vol. 49, no. 3, pp. 340-342, 2001.

[15] W. Cao, B. Zhang, A. Liu, T. Yu, D. Guo, and K. Pan, "A reconfigurable microstrip antenna with radiation pattern selectivity and polarization diversity," IEEE Antennas and Wireless Propagation Letters, vol. 11, pp. 453-456, 2012.

[16] P.-L. Chi and E. Deng, "Switchable quadri-polarization slot antenna using a single feed port," in 2017 IEEE International Symposium on Antennas and Propagation \& USNC/URSI National Radio Science Meeting. IEEE, 2017, pp. 2211-2212.

[17] K. Boonying, C. Phongcharoenpanich, and S. Kosulvit, "Polarization reconfigurable suspended antenna using rf switches and pin diodes," in The 4th Joint International Conference on Information and Communication Technology, Electronic and Electrical Engineering (JICTEE). IEEE, 2014, pp. 1-4.

[18] D. M. Pozar, Microwave engineering. John wiley \& sons, 2011.

[19] T. Infineon, PIN diode BAR64-02W Datasheet. [Online] Available: www.infineo.com. 